\title{
On Designing Observers for Time-Delay Systems with Nonlinear Disturbances
}

\author{
Zidong Wang*, D. P. Goodall and K. J. Burnham \\ Control Theory and Applications Centre \\ School of Mathematical and Information Sciences \\ Coventry University, Coventry CV1 5FB, U.K.
}

\begin{abstract}
In this paper, the observer design problem is studied for a class of time-delay nonlinear systems. The system under consideration is subject to delayed state and nonlinear disturbances. The time-delay is allowed to be time-varying, and the nonlinearities are assumed to satisfy global Lipschitz conditions. The problem addressed is the design of state observers such that, for the admissible time-delay as well as nonlinear disturbances, the dynamics of the observation error is globally exponentially stable. An effective algebraic matrix inequality approach is developed to solve the nonlinear observer design problem. Specifically, some conditions for the existence of the desired observers are derived, and an explicit expression of desired observers is given in terms of some free parameters. A simulation example is included to illustrate the practical applicability of the proposed theory.
\end{abstract}

Key Words - Algebraic matrix inequalities; Exponential stability; Nonlinear systems; Observer design; Time-delay systems.

\section{Introduction}

One of the fundamental problems in control systems is to observe the state variables of a dynamic system through available measurement. In the past three decades, this problem has attracted the attention of many researchers, see Chen (1984), O'Reilly (1983) and Unbehauen (1989). The methods that have been used in the observer design are very many, such as algebraic, geometric, inversion approaches, generalized inverse, singular value decomposition, and the Kronecker canonical form techniques. Also, different types of state observers have been extensively studied, such as reduced and minimal-order, fullorder, unknown input, functional, disturbance decoupled, etc. The application areas of the observer technique range from system monitoring, system regulation, to fault detection and isolation, see Frank (1990).

\footnotetext{
*E-mail: Zidong.Wang@coventry.ac.uk. Tel.: ++44/24 76888972, Fax: ++44/24 76888052.
} 
It has now been well recognized that the dynamic behaviour of many industrial processes contains inherent time delays. Time delays may result from the distributed nature of the system, material transport, or from the time required to measure some of the variables. It has been known that processes with time-delays are inherently difficult to control (Gorecki et al. 1989), in the sense that it is difficult to achieve satisfactory performance. Therefore, control of time-delay systems has been a subject of great practical importance that has attracted a great deal of interest for several decades, see, e.g., Niculescu et al. (1998) for an overview. It is notable that, for the observer design case, the relevant literature are relatively few for linear time-delay systems (see e.g. Lee et al. 1988, Wang and Burnham 2001, Wang et al. 1999, Wang et al. 2001 and Yao et al. 1997).

On the other hand, the problem of designing nonlinear observers has been investigated for a long time. There are mainly two research directions in this field. One is the extension of the linear Luenberger observer to the nonlinear case, such as the extended Kalman filter and the psuedo linearization technique (see Misawa and Hedrick 1989 for a survey). This technique is valid in a small range around the operating point, and often also requires heavy real-time computation (Raghavan and Hedrick 1994). The other is the differential geometric approach adopted to design exact observers for a general description of nonlinear systems ( see, for example, Hunt and Verma 1994, Xia and Zeitz 1997, and references therein), where stringent assumptions are required.

Recently, the observer/estimator design problem has been dealt with in Yaz and NaNacara (1993) for a class of nonlinear discrete-time systems. In Thau (1973), an algebraic Lyapunov equation method has been developed to tackle the observer design problem for a class of continuous-time systems with nonlinear disturbances. The results given in Thau (1973) have been useful to check the stability of the error dynamics, but have not suggested an effective approach to designing the stable observer. In Dawson et al. (1992), the problem of design observers guaranteeing global exponential stability of the observation process has been addressed for continuous-time systems with nonlinear disturbances. Moreover, by making use of the methods developed for the quadratic stabilization of uncertain systems, Raghavan and Hedrick (1994) have studied the same problem as in Thau (1973). A viable design methodology has been proposed in Raghavan and Hedrick (1994) to systematically construct the observer parameters. Unfortunately, in the literature mentioned above, the time-delay has not been taken into account. So far, to the best of the authors' knowledge, the issue of state observer design for time-delay systems with nonlinear disturbances in system states and outputs has not been fully investigated and remains to be important and challenging.

In this paper, the results of Raghavan and Hedrick (1994) and Thau (1973) are generalized to more general systems. State observers for a class of nonlinear systems with time-varying state delay are designed. The purpose is to design state observers such that the resulting observation process remains globally exponentially stable for all addressed nonlinearities. A simple, algebraic parameterized approach is exploited, which enables us to characterize both the existence conditions and the set of expected nonlinear observers for the class of nonlinear state delayed systems. It is shown that a desired solution is related to a 
Riccati-like matrix inequality (or a linear matrix inequality) that is not difficult to solve.

The remainder of the present paper is arranged as follows. The nonlinear observer design problem is formulated in Section 2 for continuous time-delay systems. In Section 3, the main results as well as detailed derivations are given, including the existence conditions and the explicit expression of the desired nonlinear observers. A simulation example is provided in Section 4 to demonstrate the validity and applicability of the proposed theory. Finally, some concluding remarks are drawn in Section 5.

Notations: The notations are quite standard. Throughout this paper, $\mathbb{R}^{n}$ and $\mathbb{R}^{n \times m}$ denote, respectively, the $n$-dimensional Euclidean space and the set of all $n \times m$ real matrices. The superscript ' $T$ ' denotes matrix transposition and the notation $X \geq Y$ (respectively, $X>Y$ ) where $X$ and $Y$ are symmetric matrices, means that $X-Y$ is positive semidefinite (respectively, positive definite). $I_{n}$ is the $n \times n$ identity matrix, $\operatorname{diag}\{\cdots\}$ stands for a block diagonal matrix. $|\cdot|$ is the Euclidean norm in $\mathbb{R}^{n}$. If $A$ is a matrix, denote by $\|A\|$ its operator norm, i.e., $\|A\|=\sup \{|A x|:|x|=1\}=\sqrt{\lambda_{\max }\left(A^{T} A\right)}$ where $\lambda_{\max }(\cdot)$ (respectively, $\lambda_{\min }(\cdot)$ ) means the largest (respectively, smallest) eigenvalue of $A$. Denote by $C\left([-h, 0] ; \mathbb{R}^{n}\right)$ the space of all continuous functions $\xi=\{\xi(\theta):-h \leq \theta \leq 0\}$ such that $\sup _{-h \leq \theta \leq 0}|\xi(\theta)|<\infty$.

\section{Problem formulation and preliminaries}

Consider the following class of nonlinear uncertain time-delay systems described by

$$
\begin{aligned}
& \dot{x}(t)=A x(t)+A_{d} x(t-h(t))+l(t, u(t), y(t))+f(t, u(t), x(t)), \\
& x(t)=\varphi(t), \quad t \in[-h, 0], \quad h=\sup _{t \in[0, \infty)} h(t),
\end{aligned}
$$

together with the measurement equation

$$
y(t)=C x(t)+g(t, u(t), x(t)),
$$

where $x(t) \in \mathbb{R}^{n}$ is the state, $u(t) \in \mathbb{R}^{m}$ is the input, $y(t) \in \mathbb{R}^{p}$ is the measurement output. $A, A_{d}, C$ are known constant matrices with appropriate dimensions. $l: \mathbb{R} \times \mathbb{R}^{m} \times \mathbb{R}^{p} \rightarrow \mathbb{R}^{n}$ is a known vector function. $f: \mathbb{R} \times \mathbb{R}^{m} \times \mathbb{R}^{n} \rightarrow \mathbb{R}^{n}$ and $g: \mathbb{R} \times \mathbb{R}^{m} \times \mathbb{R}^{n} \rightarrow \mathbb{R}^{n}$ are, respectively, the state-dependent nonlinear disturbances on the system model and on the system output. The known nonlinear vector functions $f$ and $g$ are assumed to satisfy the following global Lipschitz conditions:

$$
\begin{aligned}
& \left|f\left(t, u, x_{1}\right)-f\left(t, u, x_{2}\right)\right| \leq\left|F\left(x_{1}-x_{2}\right)\right|, \\
& \left|g\left(t, u, x_{1}\right)-g\left(t, u, x_{2}\right)\right| \leq\left|G\left(x_{1}-x_{2}\right)\right|,
\end{aligned}
$$

for all $t \in \mathbb{R}, u \in \mathbb{R}^{m}$, and $x_{1}, x_{2} \in \mathbb{R}^{n}$, where $F, G \in \mathbb{R}^{n \times n}$ are known constant matrices, and $f(t, u, \cdot)$ and $g(t, u, \cdot)$ are continuous with respect to the arguments $t$ and $u$. Also, $h(t)$ denotes the time-varying bounded state delay satisfying

$$
0 \leq h(t) \leq h<\infty, \quad \dot{h}(t) \leq d<1,
$$

where $h$ and $d$ are scalar constants. It is also assumed that the pair $(A, C)$ is detectable. 
Remark 2.1 The system (2.1)-(2.3) encompasses many important physical systems, and can be used to model many real dynamic physical processes that contain inherent time delays and nonlinear disturbances (which may result from linearization process of an originally nonlinear plant or may be an actual external nonlinear input (Raghavan and Hedrick 1994). Note that if both the state delay and the nonlinear disturbance on the system output disappear, the system (2.1)-(2.3) will reduce to that studied in Raghavan and Hedrick (1994) and Thau (1973).

In this paper, the full-order nonlinear observer under consideration is of the form

$$
\begin{aligned}
\dot{\hat{x}}(t)= & A \hat{x}(t)+A_{d} \hat{x}(t-h(t))+l(t, u(t), y(t))+f(t, u(t), \hat{x}(t)) \\
& +K[y(t)-C \hat{x}(t)-g(t, u(t), \hat{x}(t))],
\end{aligned}
$$

where $\hat{x}(t)$ is the state estimate, and $K \in \mathbb{R}^{n \times p}$ is the observer gain matrix to be designed.

Let the error state be defined by

$$
e(t):=x(t)-\hat{x}(t)
$$

then it follows from (2.1)-(2.3) and (2.7) that

$$
\begin{aligned}
\dot{e}(t)= & (A-K C) e(t)+A_{d} e(t-h(t))+[f(t, u(t), x(t))-f(t, u(t), \hat{x}(t))] \\
& -K[g(t, u(t), x(t))-g(t, u(t), \hat{x}(t))] .
\end{aligned}
$$

Now, observe the error-state system $(2.9)$ and let $e(t ; \xi)$ denote the state trajectory from the initial data $e(\theta)=\xi(\theta)$ on $-h \leq \theta \leq 0$ in $C\left([-h, 0] ; \mathbb{R}^{n}\right)$. Clearly, because of the global Lipschitz conditions on the nonlinearities $f$ and $g$, the system (2.9) admits a trivial solution $e(t ; 0) \equiv 0$ corresponding to the initial data $\xi=0$. The following stability concepts are introduced.

Definition 2.1 Consider the system (2.9) with $u: \mathbb{R} \rightarrow \mathbb{R}^{n}, x, \hat{x}: \mathbb{R} \rightarrow \mathbb{R}^{n}$ continuous and every $\xi \in C\left([-h, 0] ; \mathbb{R}^{n}\right)$, where $h$ is the upper bound of the time-delay. The trivial solution is globally asymptotically stable if: (i) the system (2.9) has global boundedness of solution; and (ii) the trivial solution is stable and satisfies

$$
\lim _{t \rightarrow \infty}|e(t ; \xi)|=0
$$

Furthermore, the trivial solution is globally exponentially stable if: (i) the system (2.9) has global boundedness of solution; and (ii) there exist constants $\alpha>0$ and $\beta>0$ such that

$$
|e(t ; \xi)| \leq \sqrt{\alpha} e^{-\beta t / 2} \sup _{-h \leq \theta \leq 0}|\xi(\theta)|
$$

The objective of this paper is to design state observers for the nonlinear time-delay system (2.1)-(2.3). More specifically, we are interested in seeking the observer gain, $K$, such that for the nonlinearities $f$ and $g$, the error dynamics of the system (2.9) remains globally exponentially stable, independent of the time-varying delay $h(t)$. 
Remark 2.2 In Wang and Burnham (2001), the filter design problem for a class of nonlinear time-delay stochastic systems was investigated. However, in Wang and Burnham $(2001), 1)$ the nonlinearities were assumed to satisfy the norm boundedness condition, which is stronger than the Lipschitz condition introduced in this paper; 2) there were no nonlinearities in the system outputs, the state delay was time-invariant, and the filter structure in Wang and Burnham (2001) was linear. Also, as will be seen later, we develop a new linear matrix inequality approach in this paper for designing the nonlinear observers that can guarantee the exponential stability of the observation process, and the algorithm can be implemented readily by using the Matlab LMI Toolbox (Gahinet et al. 1995).

\section{Main results and proofs}

The following Schur complement lemma will be needed in establishing our main results.

Lemma 3.1 (Boyd et al. 1994) Given constant matrices $\Omega_{1}, \Omega_{2}, \Omega_{3}$ where $\Omega_{1}=\Omega_{1}^{T}$ and $0<\Omega_{2}=\Omega_{2}^{T}$, then

$$
\Omega_{1}+\Omega_{3}^{T} \Omega_{2}^{-1} \Omega_{3}<0
$$

if and only if

$$
\left[\begin{array}{cc}
\Omega_{1} & \Omega_{3}^{T} \\
\Omega_{3} & -\Omega_{2}
\end{array}\right]<0
$$

or equivalently

$$
\left[\begin{array}{cc}
-\Omega_{2} & \Omega_{3} \\
\Omega_{3}^{T} & \Omega_{1}
\end{array}\right]<0 .
$$

Initially, the observer analysis problem is considered. To be specific, for a given observer, sufficient conditions are investigated for which the error dynamics of the observation process (or the 'closed-loop' system) is globally exponentially stable.

The theorem stated below will show that the global exponential stability of the error dynamics is guaranteed if a positive definite solution to a quadratic matrix inequality involving several scalar parameters is known to exist.

Theorem 3.1 Let the observer gain $K$ be given. If there exist scalars $\varepsilon_{1}, \varepsilon_{2}, \varepsilon_{3}>0$ and a symmetric matrix $P>0$ such that the following matrix inequality

$$
\begin{gathered}
(A-K C)^{T} P+P(A-K C)+P\left[\left(\varepsilon_{1}+\varepsilon_{2}^{-1}\right) I_{n}+\varepsilon_{3}^{-1} K K^{T}\right] P \\
+\frac{\varepsilon_{1}^{-1}}{1-d} A_{d}^{T} A_{d}+\varepsilon_{2} F^{T} F+\varepsilon_{3} G^{T} G<0
\end{gathered}
$$

holds, then, under the dynamics of the nonlinear time-delay error-state system (2.9), the trivial solution is globally exponentially stable. 
Proof: First for presentation convenience, the following definitions are required:

$$
\begin{aligned}
A_{c} & :=A-K C, \\
\psi(t) & :=f(t, u(t), x(t))-f(t, u(t), \hat{x}(t)), \\
\phi(t) & :=g(t, u(t), x(t))-g(t, u(t), \hat{x}(t)),
\end{aligned}
$$

and then the system (2.9) becomes

$$
\dot{e}(t)=A_{c} e(t)+A_{d} e(t-h(t))+\psi(t)-K \phi(t) .
$$

Now, an asymptotic stability property for system (2.9) is shown to hold under the condition (3.1). Consider the Lyapunov functional candidate

$$
\theta \mapsto Y(e(t+\theta))=e^{T}(t) P e(t)+\int_{t-h(t)}^{t} e^{T}(s) Q e(s) d s,
$$

where $\theta \in[-h, 0], P$ is the positive definite solution to the inequality (3.1) and $Q \geq 0$ is defined by

$$
Q:=\frac{\varepsilon_{1}^{-1}}{1-d} A_{d}^{T} A_{d}
$$

It follows that the time derivative of $Y$ along a given trajectory is governed by

$$
\begin{aligned}
\frac{d Y(e(t))}{d t}= & e^{T}(t)\left(A_{c}^{T} P+P A_{c}+Q\right) e(t)+e^{T}(t) P A_{d} e(t-h(t)) \\
& +e^{T}(t-h(t)) A_{d}^{T} P e(t)-(1-\dot{h}(t)) e^{T}(t-h(t)) Q e(t-h(t)) \\
& +\psi^{T}(t) P e(t)+e^{T}(t) P \psi(t)-\phi^{T}(t) K^{T} P e(t)-e^{T}(t) P K \phi(t) .
\end{aligned}
$$

Let $\varepsilon_{1}, \varepsilon_{2}, \varepsilon_{3}$ be positive scalars. Then the matrix inequality

$$
\left[\varepsilon_{1}^{1 / 2} e^{T}(t) P-\varepsilon_{1}^{-1 / 2} e^{T}(t-h(t)) A_{d}^{T}\right]\left[\varepsilon_{1}^{1 / 2} e^{T}(t) P-\varepsilon_{1}^{-1 / 2} e^{T}(t-h(t)) A_{d}^{T}\right]^{T} \geq 0
$$

yields

$$
\begin{aligned}
& e^{T}(t) P A_{d} e(t-h(t))+e^{T}(t-h(t)) A_{d}^{T} P e(t) \\
& \leq \varepsilon_{1} e^{T}(t) P^{2} e(t)+\varepsilon_{1}^{-1} e^{T}(t-h(t)) A_{d}^{T} A_{d} e(t-h(t))
\end{aligned}
$$

Moreover, the global Lipschitz conditions (2.4)-(2.5) and the following inequality

$$
\left[\begin{array}{c}
\varepsilon_{2}^{1 / 2} \psi(t)-\varepsilon_{2}^{-1 / 2} P e(t) \\
\varepsilon_{3}^{1 / 2} \phi(t)+\varepsilon_{3}^{-1 / 2} K^{T} P e(t)
\end{array}\right]^{T}\left[\begin{array}{c}
\varepsilon_{2}^{1 / 2} \psi(t)-\varepsilon_{2}^{-1 / 2} P e(t) \\
\varepsilon_{3}^{1 / 2} \phi(t)+\varepsilon_{3}^{-1 / 2} K^{T} P e(t)
\end{array}\right] \geq 0
$$


imply that

$$
\begin{aligned}
& \psi^{T}(t) P e(t)+e^{T}(t) P \psi(t)-\phi^{T}(t) K^{T} P e(t)-e^{T}(t) P K \phi(t) \\
\leq & \varepsilon_{2} \psi^{T}(t) \psi(t)+\varepsilon_{2}^{-1} e^{T}(t) P^{2} e(t)+\varepsilon_{3} \phi^{T}(t) \phi(t)+\varepsilon_{3}^{-1} e^{T}(t) P K K^{T} P e(t) \\
= & \varepsilon_{2} \mid f(t, u(t), x(t))-f\left(\left.(t, u(t), \hat{x}(t))\right|^{2}+\varepsilon_{3} \mid g\left((t, u(t), x(t))-g\left(\left.(t, u(t), \hat{x}(t))\right|^{2}\right.\right.\right. \\
& +\varepsilon_{2}^{-1} e^{T}(t) P^{2} e(t)+\varepsilon_{3}^{-1} e^{T}(t) P K K^{T} P e(t) \\
\leq & \varepsilon_{2}|F(x(t)-\hat{x}(t))|^{2}+\varepsilon_{3}|G(x(t)-\hat{x}(t))|^{2} \\
& +\varepsilon_{2}^{-1} e^{T}(t) P^{2} e(t)+\varepsilon_{3}^{-1} e^{T}(t) P K K^{T} P e(t) \\
= & \varepsilon_{2} e^{T}(t) F^{T} F e(t)+\varepsilon_{3} e^{T}(t) G^{T} G e(t)+\varepsilon_{2}^{-1} e^{T}(t) P^{2} e(t) \\
& +\varepsilon_{3}^{-1} e^{T}(t) P K K^{T} P e(t) \\
= & e^{T}(t)\left(\varepsilon_{2} F^{T} F+\varepsilon_{3} G^{T} G+\varepsilon_{2}^{-1} P^{2}+\varepsilon_{3}^{-1} P K K^{T} P\right) e(t) .
\end{aligned}
$$

Note that $0 \leq \dot{h}(t) \leq d<1$. Invoking (3.9), (3.11) and the definition of $Q$ in (3.7), it follows, from (3.8), that

$$
\begin{aligned}
\frac{d Y(e(t))}{d t} \leq & e^{T}(t)\left(A_{c}^{T} P+P A_{c}+Q\right) e(t)+\varepsilon_{1} e^{T}(t) P^{2} e(t) \\
& +\varepsilon_{1}^{-1} e^{T}(t-h(t)) A_{d}^{T} A_{d} e(t-h(t))-(1-d) e^{T}(t-h(t)) Q e(t-h(t)) \\
& +e^{T}(t)\left(\varepsilon_{2} F^{T} F+\varepsilon_{3} G^{T} G+\varepsilon_{2}^{-1} P^{2}+\varepsilon_{3}^{-1} P K K^{T} P\right) e(t) \\
= & e^{T}(t)\left\{A_{c}^{T} P+P A_{c}+P\left[\left(\varepsilon_{1}+\varepsilon_{2}^{-1}\right) I_{n}+\varepsilon_{3}^{-1} K K^{T}\right] P\right. \\
& \left.+\frac{\varepsilon_{1}^{-1}}{1-d} A_{d}^{T} A_{d}+\varepsilon_{2} F^{T} F+\varepsilon_{3} G^{T} G\right\} e(t)
\end{aligned}
$$

which indicates from the condition (3.1) that

$$
\frac{d Y(e(t))}{d t}<0
$$

for almost all $e(t) \neq 0$.

To this end, from Lyapunov stability theory (see, for example, Michel and Wang 1995), Theorem 6.2.22), we arrive at the conclusion that the trivial solution to the error-state system (2.9) is globally asymptotically stable for the addressed nonlinearities $f$ and $g$.

Furthermore, for the proof of the expected global exponential stability of the system (2.9), some standard manipulations on the relation (3.12) are required.

Define

$$
\begin{aligned}
\Pi:= & A_{c}^{T} P+P A_{c}+P\left[\left(\varepsilon_{1}+\varepsilon_{2}^{-1}\right) I_{n}+\varepsilon_{3}^{-1} K K^{T}\right] P \\
& +\frac{\varepsilon_{1}^{-1}}{1-d} A_{d}^{T} A_{d}+\varepsilon_{2} F^{T} F+\varepsilon_{3} G^{T} G .
\end{aligned}
$$

Let $\beta$ be the unique positive root of the equation

$$
\lambda_{\min }(-\Pi)-\beta \lambda_{\max }(P)-\beta h \lambda_{\max }(Q) e^{\beta h}=0,
$$


where $\Pi$ and $Q$ are defined, respectively, in (3.14) and (3.7), $P$ is the positive definite solution to (3.1), and $h(0 \leq h(t) \leq h)$ is the maximum of the time-varying state delay.

From (3.12), it follows that

$$
\begin{aligned}
d\left[e^{\beta t} Y(e(t), t)\right]= & e^{\beta t}[\beta Y(e(t), t) d t+d Y(e(t), t)] \\
\leq & e^{\beta t}\left(-\left[\lambda_{\min }(-\Pi)-\beta \lambda_{\max }(P)\right]|e(t)|^{2}+\right. \\
& \left.\beta \lambda_{\max }(Q) \int_{t-h(t)}^{t}|e(s)|^{2} d s\right) d t .
\end{aligned}
$$

Then, integrating both sides from 0 to $T>0$ gives

$$
\begin{aligned}
e^{\beta T} Y(e(T), T) \leq & {\left[\lambda_{\max }(P)+h(0) \lambda_{\max }(Q)\right] \sup _{-h(t) \leq \theta \leq 0}|\xi(\theta)|^{2} } \\
& -\left[\lambda_{\min }(-\Pi)-\beta \lambda_{\max }(P)\right] \int_{0}^{T} e^{\beta t}|e(t)|^{2} d t \\
& +\beta \lambda_{\max }(Q) \int_{0}^{T} e^{\beta t} \int_{t-h(t)}^{t}|e(s)|^{2} d s d t .
\end{aligned}
$$

Note that $0 \leq h(t) \leq h$ and therefore

$$
\begin{aligned}
& \int_{0}^{T} e^{\beta t} \int_{t-h(t)}^{t}|e(s)|^{2} d s d t \leq \int_{0}^{T} e^{\beta t} \int_{t-h}^{t}|e(s)|^{2} d s d t \\
\leq & \int_{-h}^{T}\left(\int_{\max (s, 0)}^{\min (s+h, T)} e^{\beta t} d t\right)|e(s)|^{2} d s \leq \int_{-h}^{T} h e^{\beta(s+h)}|e(s)|^{2} d s \\
\leq & h e^{\beta h} \int_{0}^{T} e^{\beta t}|e(t)|^{2} d t+h e^{\beta h} \int_{-h}^{0}|\xi(\theta)|^{2} d \theta .
\end{aligned}
$$

Then, in view of the definition of $\beta$ specified by (3.15), it follows that

$$
\begin{aligned}
e^{\beta T} Y(e(T), T) \leq & {\left[\lambda_{\max }(P)+h \lambda_{\max }(Q)\right] \sup _{-h \leq \theta \leq 0}|\xi(\theta)|^{2} } \\
& +\beta \lambda_{\max }(Q) h^{2} e^{\beta h} \sup _{-h \leq \theta \leq 0}|\xi(\theta)|^{2},
\end{aligned}
$$

and, hence,

$$
\begin{aligned}
|e(T)|^{2} \leq & \lambda_{\min }^{-1}(P)\left(\left[\lambda_{\max }(P)+h \lambda_{\max }(Q)\right] \sup _{-h \leq \theta \leq 0}|\xi(\theta)|^{2}\right. \\
& \left.+\beta \lambda_{\max }(Q) h^{2} e^{\beta h} \sup _{-h \leq \theta \leq 0}|\xi(\theta)|^{2}\right) e^{-\beta T} .
\end{aligned}
$$

Notice that $T>0$ is arbitrary and let

$$
\alpha:=\lambda_{\min }^{-1}(P)\left[\lambda_{\max }(P)+h \lambda_{\max }(Q)\left(1+\beta h e^{\beta h}\right)\right],
$$

the definition of global exponential stability in (2.11) is then satisfied. This completes the proof of Theorem 3.1. 
Remark 3.1 Theorem 3.1 provides a sufficient condition, in terms of a stabilty criterion, for the solvability of the original observer design problem associated with the nonlinear time-delay system (2.1)-(2.3). This result may be conservative, mainly due to the introduction of the inequalities (3.9) and (3.11). However, the conservativeness in Theorem 3.1 can be significantly reduced by appropriately selecting the design parameters $\varepsilon_{1}, \varepsilon_{2}, \varepsilon_{3}>0$. A related discussion can be seen in Xie and Soh (1994), and references therein.

Remark 3.2 Note that the stability criterion (3.1) is independent of the time-varying state delay $h(t)$, however, it does depends on the upper bound of the derivative of $h(t)$. The result is suitable for the case when the time-delay itself is unknown but the information regarding the upper bound on its derivative is available. On the other hand, if the time-delay is perfectly known, a delay-dependent criterion would be believed to be less conservative. This gives us one of the possible future research topics.

The observer synthesis problem can now be studied, that is, design an observer gain matrix $K$ such that the condition of Theorem 3.1 is satisfied. The following lemma will be required for the proof of the main results.

Lemma 3.2 Let $X \in \mathbb{R}^{m_{1} \times n_{1}}$ and $Y \in \mathbb{R}^{m_{1} \times p_{1}}\left(m_{1} \leq p_{1}\right)$. There exists a matrix $U \in$ $\mathbb{R}^{n_{1} \times p_{1}}$ which simultaneously satisfies $Y=X U$ and $U U^{T}=I$ if and only if $X X^{T}=Y Y^{T}$.

Proof: See Glover (1984) for a proof of this lemma.

For the sake of simplicity, define

$$
\Gamma\left(\varepsilon_{1}, \varepsilon_{2}, \varepsilon_{3}, P\right)=A^{T} P+P A+\left(\varepsilon_{1}+\varepsilon_{2}^{-1}\right) P^{2}+\frac{\varepsilon_{1}^{-1}}{1-d} A_{d}^{T} A_{d}+\varepsilon_{2} F^{T} F+\varepsilon_{3} G^{T} G .
$$

The next theorem establishes the necessary and sufficient conditions for the existence of an observer gain, $K$, satisfying the condition (3.1).

Theorem 3.2 There exist positive scalars $\varepsilon_{1}, \varepsilon_{2}, \varepsilon_{3}$ and a symmetric positive definite matrix $P$ such that the matrix inequality (3.1) has a solution $K$ if and only if the following quadratic matrix inequality

$$
\varepsilon_{3} C^{T} C-\Gamma\left(\varepsilon_{1}, \varepsilon_{2}, \varepsilon_{3}, P\right)>0
$$

holds. Furthermore, if (3.17) is true, all matrices $K$ satisfying the matrix inequality (3.1) can be parameterized by

$$
K=\varepsilon_{3} P^{-1} C^{T}+\varepsilon_{3}^{1 / 2} P^{-1} \Lambda U,
$$

where $\Lambda \in \mathbb{R}^{n \times p}$ is any matrix satisfying

$$
\Lambda \Lambda^{T}<\varepsilon_{3} C^{T} C-\Gamma\left(\varepsilon_{1}, \varepsilon_{2}, \varepsilon_{3}, P\right)
$$

and $U \in \mathbb{R}^{p \times p}$ is an arbitrary orthogonal matrix (i.e., $U U^{T}=I_{n}$ ). 
Proof: It is straightforward to rewrite the matrix inequality (3.1) as

$$
-C^{T} K^{T} P-P K C+\varepsilon_{3}^{-1} P K K^{T} P+\Gamma\left(\varepsilon_{1}, \varepsilon_{2}, \varepsilon_{3}, P\right)<0,
$$

or, alternatively,

$$
\left[\varepsilon_{3}^{-1 / 2} P K-\varepsilon_{3}^{1 / 2} C^{T}\right]\left[\varepsilon_{3}^{-1 / 2} P K-\varepsilon_{3}^{1 / 2} C^{T}\right]^{T}<\varepsilon_{3} C^{T} C-\Gamma\left(\varepsilon_{1}, \varepsilon_{2}, \varepsilon_{3}, P\right) .
$$

It is apparent that there exists an observer gain matrix $K$ such that the inequality (3.1) (or equivalently (3.21)) holds for some positive scalars $\varepsilon_{1}, \varepsilon_{2}, \varepsilon_{3}$ and a positive definite matrix $P$ if and only if the right-hand side of (3.21) is positive definite, i.e., (3.17) holds. Therefore, the proof of the first part of this theorem is concluded.

Assume that (3.17) is satisfied. Note that the dimension of the observer gain $K$ is $n \times p$ and $p \leq n$. From (3.21) and the definition of $\Lambda \in \mathbb{R}^{n \times p}$ (specified in (3.19)), it follows that there exists $\Lambda$ such that

$$
\left[\varepsilon_{3}^{-1 / 2} P K-\varepsilon_{3}^{1 / 2} C^{T}\right]\left[\varepsilon_{3}^{-1 / 2} P K-\varepsilon_{3}^{1 / 2} C^{T}\right]^{T}=\Lambda \Lambda^{T} .
$$

Thus, Lemma 3.2 implies that

$$
\varepsilon_{3}^{-1 / 2} P K-\varepsilon_{3}^{1 / 2} C^{T}=\Lambda U
$$

where $U \in \mathbb{R}^{p \times p}$ is an arbitrary orthogonal matrix. Therefore, the expression (3.18) follows immediately. This completes the proof of the theorem.

Remark 3.3 In practical applications, it is very desirable to directly solve the Quadratic Matrix Inequality (QMI) (3.17), and then obtain the expected observer gain readily from (3.18). When working with the QMI (3.17), the local numerical searching algorithms suggested in Beran and Grigoriadis (1996) and Geromel et al. (1993) are very effective for a relatively low-order model. A related discussion of the solution algorithms for QMIs can also be found in Saberi et al. (1995).

For relatively high-order model, the aforementioned algorithms no longer work well. Fortunately, we could transform the QMI (3.17) into an associated linear matrix inequality (LMI). It should be pointed out that, since LMIs intrinsically reflect constraints rather than optimality, they tend to offer more flexibility for combining several constraints. LMIs can now be solved efficiently via interior-point optimization algorithms, such as those described in Gahinet et al. (1995). Moreover, software like MATLAB LMI Toolbox are now available to solve such LMIs efficiently.

The following corollary gives an LMI representation of Theorem 3.2.

Corollary 3.1 If there exist positive scalars $\varepsilon_{1}, \varepsilon_{2}, \varepsilon_{3}$ and a symmetric positive definite 
matrix $P$ such that the following linear matrix inequality

$$
\left[\begin{array}{ccccc}
A^{T} P+P A+\varepsilon_{3}\left(G^{T} G-C^{T} C\right) & P & P & \varepsilon_{1}^{-1}(1-d)^{-1 / 2} A_{d}^{T} & \varepsilon_{2} F^{T} \\
P & -\varepsilon_{1}^{-1} I & 0 & 0 & 0 \\
P & 0 & -\varepsilon_{2} I & 0 & 0 \\
\varepsilon_{1}^{-1}(1-d)^{-1 / 2} A_{d} & 0 & 0 & -\varepsilon_{1}^{-1} I & 0 \\
\varepsilon_{2} F & 0 & 0 & 0 & -\varepsilon_{2} I
\end{array}\right]<0
$$

which is linear on the parameters $\varepsilon_{1}^{-1}, \varepsilon_{2}, \varepsilon_{3}$, and $P$, holds, then all matrices $K$ satisfying the matrix inequality (3.1) can be parameterized by (3.18), where $\Lambda \in \mathbb{R}^{n \times p}$ is any matrix satisfying (3.19), and $U \in \mathbb{R}^{p \times p}$ is an arbitrary orthogonal matrix (i.e., $U U^{T}=I_{n}$ ).

Proof: In view of Theorem 3.2, it suffices to show that, there exist $\varepsilon_{1}>0, \varepsilon_{2}>0, \varepsilon_{3}>0$ and $P>0$ such that (3.17) holds if and only if there exist $\varepsilon_{1}>0, \varepsilon_{2}>0, \varepsilon_{3}>0$ and $P>0$ such that (3.24) holds.

It is easy to rearrange (3.17) as follows:

$$
A^{T} P+P A+\varepsilon_{3}\left(G^{T} G-C^{T} C\right)+\Xi \Xi^{T}<0
$$

where

$$
\Xi:=\left[\begin{array}{llll}
\varepsilon_{1}^{1 / 2} P & \varepsilon_{2}^{-1 / 2} P & \varepsilon_{1}^{-1 / 2}(1-d)^{-1 / 2} A_{d}^{T} & \varepsilon_{2}^{1 / 2} F^{T}
\end{array}\right] .
$$

If follows from the Schur Complement Lemma (Lemma 3.1) that, the above inequality holds if and only if

$$
\left[\begin{array}{ccccc}
A^{T} P+P A+\varepsilon_{3}\left(G^{T} G-C^{T} C\right) & \varepsilon_{1}^{1 / 2} P & \varepsilon_{2}^{-1 / 2} P & \varepsilon_{1}^{-1 / 2}(1-d)^{-1 / 2} A_{d}^{T} & \varepsilon_{2}^{1 / 2} F^{T} \\
\varepsilon_{1}^{1 / 2} P & -I & 0 & 0 & 0 \\
\varepsilon_{2}^{-1 / 2} P & 0 & -I & 0 & 0 \\
\varepsilon_{1}^{-1 / 2}(1-d)^{-1 / 2} A_{d} & 0 & 0 & -I & 0 \\
\varepsilon_{2}^{1 / 2} F & 0 & 0 & 0 & -I
\end{array}\right]<0 .
$$

Pre- and post-multiplying the inequality (3.26) by the matrix

$$
\left[\begin{array}{ccccc}
I & 0 & 0 & 0 & 0 \\
0 & \varepsilon_{1}^{-1 / 2} I & 0 & 0 & 0 \\
0 & 0 & \varepsilon_{2}^{1 / 2} I & 0 & 0 \\
0 & 0 & 0 & \varepsilon_{1}^{-1 / 2} I & 0 \\
0 & 0 & 0 & 0 & \varepsilon_{2}^{1 / 2} I
\end{array}\right]
$$

yield (3.24), and the proof is complete.

The following result, which is easily accessible from Theorem 3.1 and Theorem 3.2, solves the observer design problem addressed in this paper for the nonlinear time-delay system (2.1)-(2.3). 
Theorem 3.3 Consider the nonlinear time-delay system (2.1)-(2.3) and the associated full-order observer (2.7). If there exist positive scalars $\varepsilon_{1}, \varepsilon_{2}, \varepsilon_{3}$ and a symmetric positive definite matrix P such that the QMI (3.17) or LMI (3.24) holds, then the observer (2.7) with its parameter given in (3.18) will be such that, under the dynamics of the observation error (i.e., the solution of the error-state system (2.9)), the error zero-state is globally exponentially stable.

Remark 3.4 It is worth mentioning that, there is a lot of freedom (such as the choices of matrices $\Lambda$ and $U$ ) in the observer design that may be used to improve other system properties. One of the future research topics is how to exploit such freedom to achieve the specified reliable constraint on the observation process.

\section{Numerical simulation}

To illustrate the usefulness and flexibility of the proposed theory, a numerical simulation example is discussed in this section.

Assume that the nonlinear state delayed system (2.1)-(2.3) is described by the following data

$$
\begin{gathered}
A=\left[\begin{array}{ccc}
-1.8 & 0.2 & -0.5 \\
-0.3 & -2.6 & 0.9 \\
-0.3 & 0.7 & -2.4
\end{array}\right], A_{d}=\left[\begin{array}{ccc}
0.04 & -0.01 & -0.01 \\
0.01 & -0.03 & 0.02 \\
0.01 & -0.01 & 0.05
\end{array}\right] \\
C=I_{3}, \quad h(t)=0.4 \sin (t)(d=0.4), \quad l(t, u(t), y(t)) \equiv 0
\end{gathered}
$$

For simulation purposes, suppose that

$$
f(t, u, x)=\left[\begin{array}{c}
0.5 \cos \left(x_{2}+x_{3}\right) \\
0 \\
-0.6 \cos \left(x_{1}-x_{2}\right)
\end{array}\right], g(t, u, x)=\left[\begin{array}{c}
0.4 \cos x_{2} \\
-0.6 \cos x_{1} \\
0.5 \sin x_{3}
\end{array}\right] .
$$

Our aim is to design an observer, with the structure (2.7), for the nonlinear time-delay system (2.1)-(2.3), such that, under the dynamics of the error system, the error zero-state is globally exponentially stable.

First, $F$ and $G$ are estimated to be $F=0.5 I_{3}$ and $G=0.4 I_{3}$. Then, solve the LMI (3.24) to give:

$$
\varepsilon_{1}=0.3012, \varepsilon_{2}=1.4896, \varepsilon_{3}=0.8001, P=\left[\begin{array}{ccc}
3.1083 & 0.3707 & 0.8304 \\
0.3707 & 4.7192 & -1.9180 \\
0.8304 & -1.9180 & 4.5559
\end{array}\right] \text {. }
$$

It follows from Corollary 3.1 that, the desired observer gain can be parameterized by $K=\varepsilon_{3} P^{-1} C^{T}+\varepsilon_{3}^{1 / 2} P^{-1} \Lambda U$, where $\Lambda \in \mathbb{R}^{3 \times 3}$ and $U \in \mathbb{R}^{3 \times 3}$ satisfy

$$
\Lambda \Lambda^{T}<\left[\begin{array}{ccc}
2.8001 & -0.0000 & 0.0000 \\
0.0000 & 2.8001 & 0.0000 \\
-0.0000 & -0.0000 & 2.8001
\end{array}\right], U U^{T}=I_{3}
$$


Due to the freedom in choosing the parameters $\Lambda$ and $U$, the number of the desired observer gains is infinite. Thus, to illustrate such design flexibility, we take the following four cases as examples:

Case 1: $\Lambda=1.5 I_{3}, U=I_{3}$;

Case 2: $\Lambda=-1.5 I_{3}, U=\operatorname{diag}\{-1,-1,1\}$;

Case 3: $\Lambda=0.45 I_{3}, U=\operatorname{diag}\{1,-1,-1\}$;

Case 4: $\Lambda=-0.45 I_{3}, U=-I_{3}$.

For the above four cases, the corresponding observer gain matrices are obtained from (3.18), respectively, as follows:

$$
\begin{aligned}
& \text { Case 1: } \quad K=\left[\begin{array}{ccc}
0.7583 & -0.1396 & -0.1970 \\
-0.1396 & 0.5732 & 0.2668 \\
-0.1970 & 0.2668 & 0.6183
\end{array}\right], \\
& \text { Case 2: } \quad K=\left[\begin{array}{ccc}
0.4100 & -0.0755 & -0.0407 \\
-0.0755 & 0.3099 & 0.0551 \\
-0.1065 & 0.1442 & 0.1277
\end{array}\right], \\
& \text { Case 3: } \quad K=\left[\begin{array}{ccc}
0.4258 & -0.0259 & -0.0366 \\
-0.0784 & 0.1064 & 0.0495 \\
-0.1106 & 0.0495 & 0.1148
\end{array}\right], \\
& \text { Case 4: } \quad K=\left[\begin{array}{ccc}
0.4258 & -0.0784 & -0.1106 \\
-0.0784 & 0.3219 & 0.1498 \\
-0.1106 & 0.1498 & 0.3472
\end{array}\right] .
\end{aligned}
$$

Denote the error states $e_{i}=x_{i}-\hat{x}_{i}(i=1,2,3)$. In the four cases, the responses of error dynamics to initial conditions are shown, respectively, in Figures 1-4. The simulation results confirm that the desired goal has been achieved.

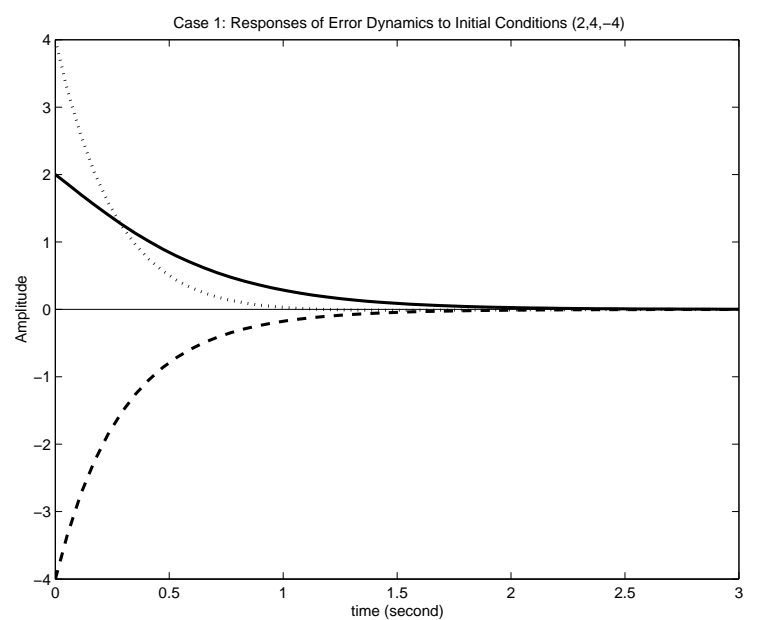

Figure 1: $e_{1}$ (solid), $e_{2}$ (point), $e_{3}$ (dashed).

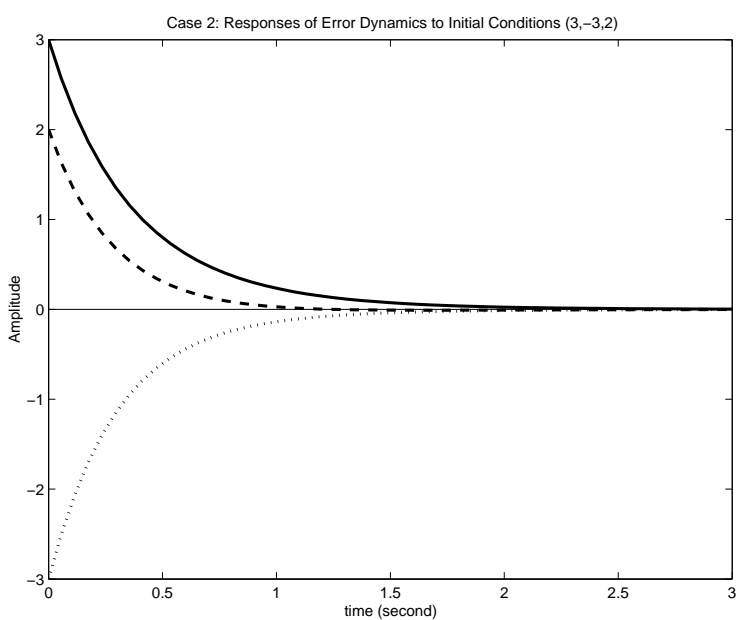

Figure 2: $e_{1}$ (solid), $e_{2}$ (point), $e_{3}$ (dashed). 


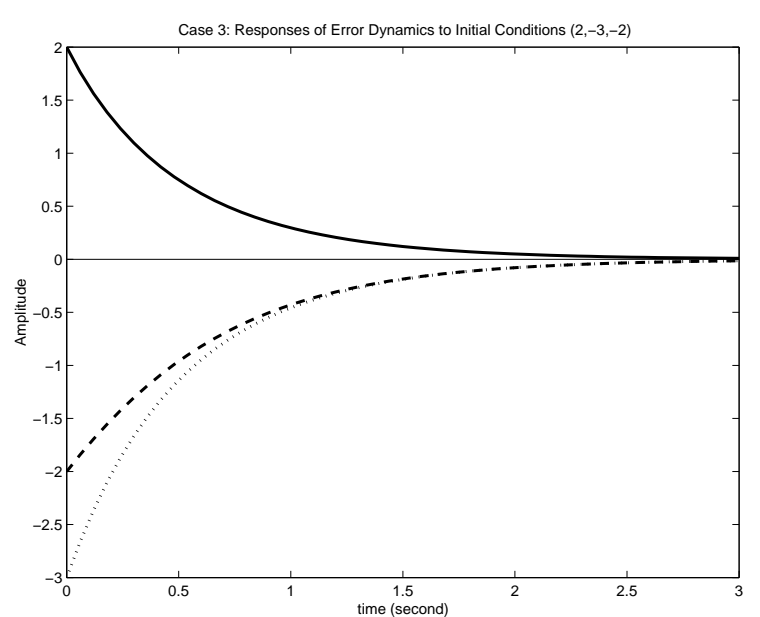

Figure 3: $e_{1}$ (solid), $e_{2}$ (point), $e_{3}$ (dashed).

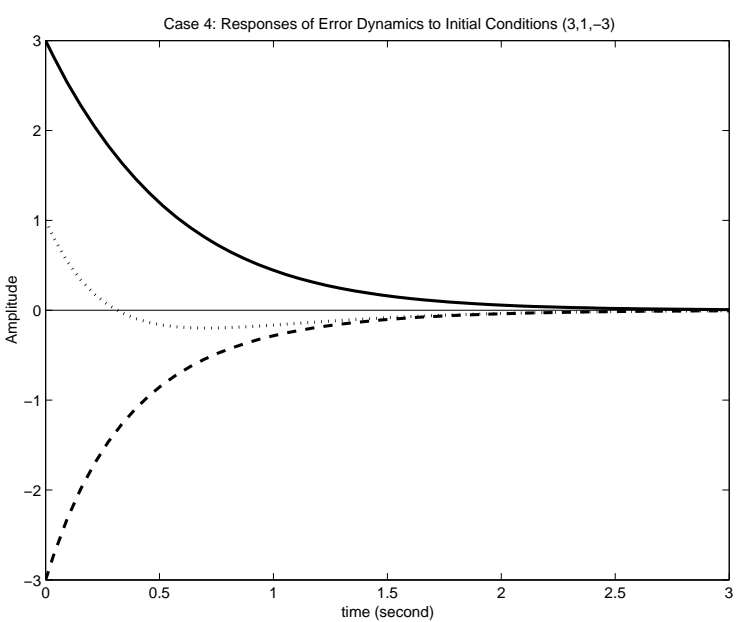

Figure 4: $e_{1}$ (solid), $e_{2}$ (point), $e_{3}$ (dashed).

Remark 4.1 As can be seen in the numerical examples, the desired solution set, if not empty, must be very large. In other words, every observer gain, which belongs to the desired solution set, could make the corresponding observation system exponentially stable. In this sense, we could think there are no essential 'differences' between different observer gains in the desired solution set, since they produce the 'same' performance, i.e., the exponential stability of estimation error dynamics. The remaining design flexibility makes it possible to enforce further expected requirements on the observation process, such as robustness against parameter uncertainties.

\section{Conclusions}

This paper has dealt with the problem of nonlinear state observer design for a class of continuous-time systems with time-varying state delay. A full-order nonlinear observer structure has been adopted. A quadratic matrix inequality (QMI) approach, or a linear matrix inequality (LMI) approach, has been developed to solve the problem addressed. Specifically, the conditions for the existence of the expected nonlinear observers have been derived in terms of the positive definite solution to an QMI (or LMI) involving several scalar parameters. An analytical expression, characterizing the desired observers, has been obtained, and a simulation example has shown the usefulness of the proposed design approach. In particular, it has been demonstrated that the desired nonlinear observers of time-delay systems are usually a large set in terms of some free parameters. The resulting freedom can be used to meet other expected performance requirements, such as the constraints on the $H_{\infty}$ norm of the transfer function from possible noise input to observation error output. The main results can also be extended to discrete-time systems and sampled-data systems. These will be the subjects of further investigations. 


\section{Acknowledgements}

The authors would like to thank three anonymous referees for their valuable comments that have improved the presentation. Z. Wang is grateful to Professor H. Unbehauen of Ruhr-University Bochum of Germany and Professor D. Prätzel-Wolters of University of Kaiserslautern of Germany for their useful suggestions, to Dr. J. Lam of The University of Hong Kong and Dr. D. W. C. Ho of City University of Hong Kong for several helpful discussions. The work of Z. Wang was supported in part by the University of Kaiserslautern of Germany and the Alexander von Humboldt Foundation of Germany.

\section{References}

Beran, E. and Grigoriadis, K., 1996, A combined alternating projections and semidefinite programming algorithm for low-order control design, Prep. 13th IFAC World Congress, San Francisco, CA, USA, C: 85-90.

S. Boyd, L. El Ghaoui, E. Feron, V. Balakrishnan, Linear matrix inequalities in system and control theory, Studies in Applied Mathematics, Philadelphia, PA: SIAM, 1994.

Chen, C. -T., 1984, Linear system theory and design. Holt, Rinehart, and Winston, New York, USA.

Dawson, D. M., Qu, Z. and Carroll, J. C., 1992, On the state observation and output feedback problems for nonlinear uncertain dynamic systems, Systems \& Control Letters, 18: 217-222.

Furuta, K., Hara, S. and Mori, S., 1976, A class of systems with the same observer, IEEE Trans. Automat. Contr., 21: 572-576.

Frank, P. M., 1990, Fault diagnosis in dynamic systems using analytical and knowledge-based redundancy - a survey and some new results, Automatica, 26: 459 - 474.

Gahinet, P., Nemirovsky, A., Laub, A. J. and Chilali, M., 1995, LMI control toolbox: for use with Matlab, The MATH Works Inc., U.S.A.

Geromel, J. C., Peres, P. L. D. and Souza, S. R., 1993, Output feedback stabilization of uncertain systems through a min/max problem, Prep. 12th IFAC World Congress, Sydney, Australia, 6: 35-38.

Glover, K., 1984, All optimal Hankel-norm approximations of linear multivariable systems and their $L^{\infty}$-error bounds, Int. J. Control, 39: 1115-1193.

Gorecki, H., Fuksa, S., Grabowski, P. and Korytowski, A., 1989, Analysis and synthesis of time delay systems, John Wiley \& Sons, New York, U.S.A.

Hunt, L. R. and Verma, M., 1994, Observers for nonlinear systems in steady state, IEEE Trans. Automat. Contr., 39: 2113-2118.

Lee, S. J., Kwon, W. H. and Kim, S. W., 1988, LQG/LTR methods for linear input-delayed systems, Int. J. Contr., 47: 1179-1194.

Michel, A. N. and Wang, K., 1995, Qualitative theory of dynamical systems. Marcel Dekker, New York, USA.

Misawa, E. A. and Hedrick, J. K., 1989, Nonlinear observers: a state-of-the-art survey, Trans. ASME: J. Dynamic Systems, Measurement, and Control, 111: 344-532.

Niculescu, S. -I., Verriest, E. I., Dugard, L. and Dion, J. M., 1998, Stability and robust stability of time-delay systems: a guided tour, Dugard, L. (ed.) et al., Stability and control of time-delay systems, Springer, Berlin, Germany, Lect. Notes Control Inf. Sci., 228, 1-71.

O’Reilly, J., 1983, Observer for linear systems. Academic Press, New York, USA. 
Petersen, I. R. and McFarlane, D. C., 1994, Optimal guaranteed cost control and filtering for uncertain linear systems, IEEE Trans. Automat. Contr., 39: 1971-1977.

Raghavan, S. and Hedrick, J. K., 1994, Observer design for a class of nonlinear systems, Int. J. Contr., 59: $515-528$.

Saberi, A., Sannuti, P and Chen, B. M., 1995, $\mathrm{H}_{2}$ optimal control, Prentice Hall International, Series in Systems and Control Engineering, London, U.K.

Thau, F. E., 1973, Observing the state of nonlinear dynamic systems, Int. J. Contr., 17: 471-479.

Unbehauen, H., 1989, Regelungstechnik II, Vieweg Verlag, Braunschweig, Germany.

Wang, Z. and Burnham, K. J., 2001, Robust filtering for a class of stochastic uncertain nonlinear time-delay systems via exponential state estimation, IEEE Transactions on Signal Processing, 49: $794-804$.

Wang, Z., Huang, B. and Unbehauen, H., 1999, Robust $H_{\infty}$ observer design of linear state delayed systems with parametric uncertainty: the discrete-time case, Automatica, 35: 1161-1167.

Wang, Z., Huang, B. and Unbehauen, H., 2001, Robust $H_{\infty}$ observer design of linear time-delay systems with parametric uncertainty, Systems \& Control Letters, 42: 303-312.

Xia, X. and Zeitz, M., 1997, On nonlinear continuous observers, Int. J. Contr., 66: 943-954.

Xie, L. and Soh, Y. C., 1994, Robust Kalman filtering for uncertain systems, Systems \& Control Letters. 22: 123-129.

Yaz, E. and NaNacara, W., 1993, Nonlinear estimation by covariance assignment, Prep. 12th IFAC World Congress, Sydney, Australia, 6: 87-90.

Yao, Y. X., Zhang, Y. M. and Kovacevic, R., 1997, Functional observer and state feedback for input time-delay systems, Int. J. Contr., 66: 603-617. 